\title{
A Stable Routing Protocol based on DSR Protocol for Mobile Ad Hoc Networks
}

\author{
Golsum Najafi ${ }^{\mathrm{a}}$, Sajjad Jahanbakhsh Gudakahriz ${ }^{\mathrm{b}}$ \\ ${ }^{a, b}$ Department of Computer Engineering, Germi Branch, Islamic Azad University, Germi, Iran
}

Received: 13 October 2017; Accepted: 13 February 2018; Published: 08 May 2018

\begin{abstract}
Mobile ad hoc network is a wireless non-centralized network. This network includes a set of distributed nodes with any base or central management, develop a temporary network. Such a way, routing is of the basic challenges of these kinds of networks. Till now, various protocols have been provided for routing in these kinds of networks. One of the optimum routing techniques in these networks is stable routing. In the stable routing, the target is to use paths for send and receive that probably have a higher life period. This causes the data to be sent from a more confident path and the need to new routing will be less, due to liquidation of the current path. In this paper, the target is to provide a stable routing protocol with high efficiency for these kinds of networks, by improving the DSR routing protocol. In the provided protocol, beside the path stability, the energy of the path nodes and path length will be considered too, in order to discover a path with higher quality and use it. The provided protocol will be called as ST-DSR. The result of stimulation in the NS-2 environment shows that the ST-DSR has a better operation toward the base protocol, meaning DSR.
\end{abstract}

Index Terms: Mobile ad hoc networks, Routing, Stable routing, DSR protocol.

(C) 2018 Published by MECS Publisher. Selection and/or peer review under responsibility of the Research Association of Modern Education and Computer Science

\section{Introduction}

Nowadays, due to the increase of the wireless communications and the need of its utilization in different areas, the establishment and utilization of the wireless networks have had a considerable growth. In this way, mobile ad hoc networks, also, due to having benefits including the lack of needing predetermined base, central management and also mobility and high flexibility have been taken into account [1,2]. Mobile ad hoc networks are a non-centralized wireless network, where a network node, sends data for the next nodes and decision

* Corresponding author.

E-mail address: 
taking for sending data for the next node is dynamically conducted for that node. Mobile case network is a centralized wireless network. This network includes a set of distributed nodes that form a temporary network without any base or central management $[3,4,5]$. In these networks, there is no base, like a router or access point, however nodes are connected directly and each node, participates in routing by sending data for other nodes. In the case networks, nodes can act as router and host $[6,7,8]$. Due to the instability of mobile ad hoc networks, the issue of discovering route maintenance has a particular importance. Despite the many recent advances in the development of efficient routing protocols that can efficiently operate in an environment of case networks with a high number of mobile nodes, is also of the challenges of this network. Routing in the case networks, is the task of available nodes in the network, meaning that, there is no secondary reticular device like switch, router or hub for routing, but these nodes form the network that conducts the routing operation, too. Routing protocols in this network is difficult due to the random movement of any node. That means, a path is optimum in a time, may even not exist in seconds later. So, routing in the routing networks with mobile offset has fundamental challenges and optimum methods should be taken into account in these networks. One of the most important issues that should be considered in any kind of network is routing and discovering optimum paths from any offset to any destination. Routing in the wireless networks with frameworks that access point is constant in the, is a hard and important problem and is needy of special plans and solutions. Solving these problems in the offset routing networks that the nodes are not constant and are frequently changing their positions is so difficult and needs more preparations, because these kinds of networks have no constant topology and in every moment, the picking of the nodes have changes $[9,10,11]$.

Routing in the mobile ad hoc networks, can be conducted in different ways, which is dependent of the routing strategy and network structure. According to the routing strategy, routing protocols conclude of two parts: Table-driven routing and On-demand routing protocols. Table-driven routing protocols, maintain the routing information, even before it is needed, so these protocol are called Proactive routing protocols, too, where the provided protocols of this group is DSDV [12]. On-demand routing protocols, do not maintain routing information, if there is no connection, so, these protocols, are called reactive routing protocols, too. The provided protocols of this group are DSR [13], AODV [14] and TORA [15].

In this paper, the target is providing a stable protocol with a high efficiency for routing in the mobile ad hoc networks. The provided protocol is based on the DSR protocol. For this purpose, in section 2, we briefly review related works. We will introduce DSR protocol in the section 2. In section 3, the provided protocol will be introduced in detail. In the section 4, the stimulation is conducted and the results are explained and finally in section 5 we will have a conclusion of the subject.

\section{Related Works}

In recent years many works have done on routing protocol in mobile ad hoc networks. In the following we review some of this works related with our work. In [16] discussed on an energy aware routing protocol that uses zone based routing with parallel collision guided broadcasting protocol (ZCG) that uses parallel and distributed broadcasting technique to reduce redundant broadcasting and to accelerate the path discovery process, while maintaining high reach ability ratio as well as keeping node energy consumption low. Meantime, to reduce energy consumption, some nodes may choose for routing misbehavior such that they take part in routes finding process but do not forward data packets. Detecting and mitigating routing misbehavior, forcing malicious nodes to cooperate, and extending lifetime of the network should be considered in routing decisions. In [17] proposed a Power Aware Cooperation Enforcement (PACE) distributed mechanism to help nodes make intelligent routing and forwarding decisions. In MANET, network topology is dynamic and node positions change continual. An effective mechanism for route discovery in such networks is broadcasting, but routing overhead associated with broadcasting can be large in high dynamic networks. To reduce this routing overhead in MANETs, neighbor coverage based probabilistic rebroadcast protocol is used. To determine the rebroadcast order, a novel rebroadcast delay is used and it obtains the more accurate additional coverage ratio by sensing neighbor coverage knowledge. The rebroadcast probability is calculated by merging the additional coverage 
ratio and connectivity factor. In [18] method Energy Efficient Neighbor Coverage Protocol (EENCP) is introduced. In [19] energy consumption in MANET is further optimized by using a hardware circuit called the Remote Activated Switch (RAS) to wake up sleeping nodes. These energy-saving approaches are wellestablished in reactive protocols. However, there are certain issues to be addressed when using EASRP in a hybrid protocol, especially a proactive protocol. Real-time multimedia applications in MANETs make Quality of Service $(Q o S)$ support, a crucial issue regarding terms of increased throughput, reduced jitter, and available bandwidth. Most of the existing routing protocols focus on straight forward hop-count metric and are less adaptable to densely distributed and highly mobile networks. Therefore, Multicast communication with $Q o S$ in densely populated highly dynamic network an exciting research challenges. The objective of [20] is to introduce a $Q o S$ aware routing metric that determines a reliable forwarding node based on Link Stability cost Function $(L S F)$.

\section{DSR Protocol}

Dynamic Source Routing Protocol is one of the demand-driven routing protocols based on resource routing. Additionally, nodes do not need to distribute their routing tables to the neighboring nodes, which save a lot of network bandwidth. Another important issue of the DSR protocol is that whenever there is a failure link, the RERR packet is released to the original source, which initiates the path detection process at the new turn. The link cannot be restored locally [21,22]. Two main phases are considered for this protocol: route detection and path updates. The route discovery phase uses the packets of request and response, and the route update phase uses acknowledgments and link errors. The DSR protocol is a proactive protocol that can manage source routing networks without the need for routing tables and updating them. In the DSR protocol, the sender specifies all of the source paths to the destination and stores all the middle nodes in the packets. This protocol operates on the basis of link state algorithms, meaning each node can store the best route to the destination. Also, if a change occurs in the network, all network nodes are notified through the general flooding of this change. The DSR protocol includes two major mechanisms. In this type of mobile nodes, you need to provide drafts for the routes, they are aware of. DSR protocol is a loop-based, source-driven and demand-driven protocol. The DSR protocol is the most appropriate routing protocol for small and small networks. Of course, due to the features that the protocol holds in the paths stored in its memory, it can also perform well in large and high-traffic networks [23, 24].

\section{Proposed protocol}

The DSR routing protocol is one of the promising routing protocols in the mobile source routing network that has been dealt with in many articles and researches and is being used as an indicator for measuring the performance of new protocols. In the DSR routing protocol, the shortest route is used to select the best route, which is not optimal, since the shortest path is not necessarily the best route. So, in this study, by modifying this protocol and designing and implementing a system with the new idea of the optimal protocol of ST-DSR, the use of this method has increased the efficiency of the new protocol relative to the DSR routing protocol. Increasing network throughput and packet delivery rates, as well as reducing end-to-end latency and reducing network power consumption are among the results.

The main idea behind the protocols is to discover and use a more stable and high-quality path in the DSR protocol. Such a way that when paths are discovered, the routes between the source and destination nodes are evaluated in terms of the stability of the path links, the energy of the route nodes and the length of the path using Formula 1, and a path is discovered that according to This formula has the highest stability and quality

$$
\text { Formula (1) } R Q=\frac{\min \_t t l \times \text { min_energy }}{\text { Hopcount }}
$$


In this formula, min_ttl is minimum time to live of links in the path, min_energy is minimum energy of the path nodes and Hopcount is path length.

Let's explain the details of the provided protocol with an example. Figure 1 shows a hypothetical mobile network. In this form, the values on the nodes represent the percentage of energy remaining in the node and the numbers on the links represent the lifetime of each link. In this network, node S wants to communicate with node $\mathrm{D}$ at first, the S-node sends packets of the request to the destination. When request packets move toward the destination, the shortest link life between the links in each path, the lowest node energy is considered between the nodes in the path and the length of each path. In the hypothetical network, packet requests for the route are traversed from the 3 paths $\mathrm{S}, \mathrm{N} 1, \mathrm{~N} 2, \mathrm{~N} 3, \mathrm{D} ; \mathrm{S}, \mathrm{N} 4, \mathrm{~N} 5, \mathrm{D} ; \mathrm{S}, \mathrm{N} 6, \mathrm{~N} 7, \mathrm{~N} 8, \mathrm{D}$; and arrive at the destination node. The node $\mathrm{D}$ evaluates the quality of these three paths using Formula 1, which for the first path is $R Q=2.5$, for the second path $R Q=0.33$ and for the third path $R Q=0.75$. This way, because the route has the highest stability and quality, this route is selected and the packet response to the route is sent from this path. This way, this path is discovered and used.

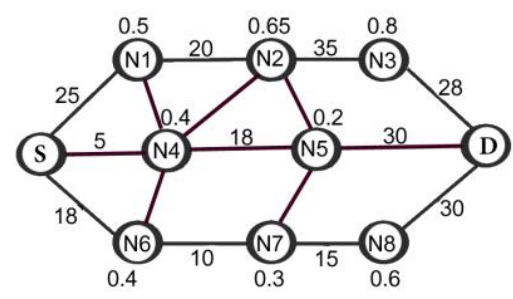

Fig.1. ST-DSR protocol process

\section{Simulation and evaluation of the proposed protocol}

In order to evaluate the proposed method, the changes described above apply in the NS-2 simulator on the DSR protocol and under different scenarios in terms of some of the most important routing parameters such as packet delivery rates, end-to-end latency, and operational power compare the DSR protocol. The comparison between new protocol and base protocol has done on the following efficiency criteria: Throughput, End-to-end delay and Route discovery frequency.

\subsection{First simulation scenario}

In this scenario, the time to stop the nodes is considered variable. The simulation parameters are shown in Table 1. The results for this scenario are shown in Figures 2 to 4.

Table. 1 First simulation scenario parameters

\begin{tabular}{|c|c|}
\hline Parameters & Parameter values \\
\hline Simulation space & $500 \times 500 \mathrm{~m}^{2}$ \\
\hline Simulation time & 500 Second \\
\hline Routing protocol & DSR and ST-DSR \\
\hline Nodes pause time & $20,40,60,80,100$ Second \\
\hline Nodes speed & $20 \mathrm{~m} / \mathrm{s}$ \\
\hline Nodes number & 75 \\
\hline IFQ & Drop tail \\
\hline Queue capacity & 50 packets \\
\hline MAC layer & $802.11 \mathrm{~b}$ \\
\hline
\end{tabular}




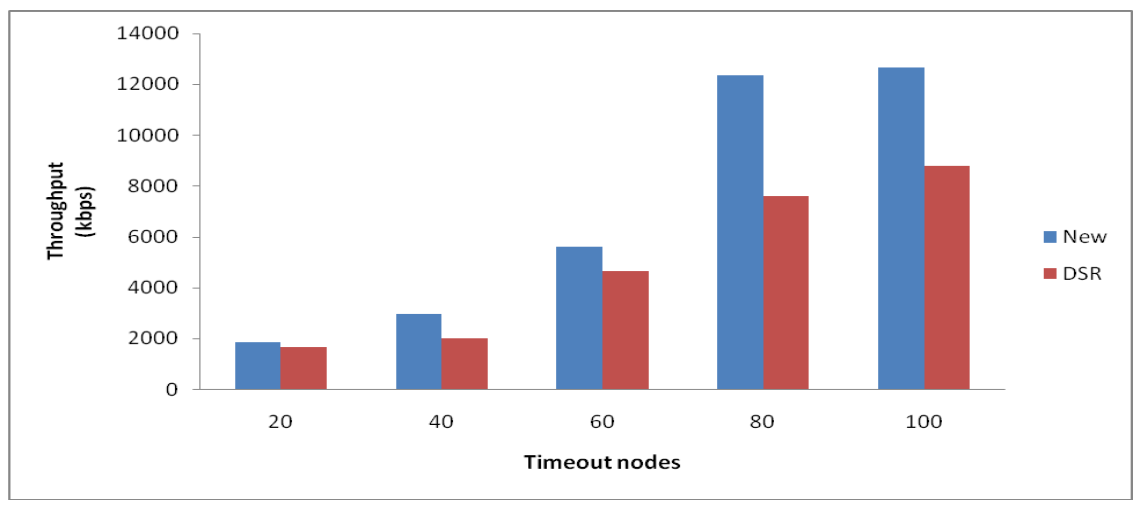

Fig.2. Average delay versus pause time nodes

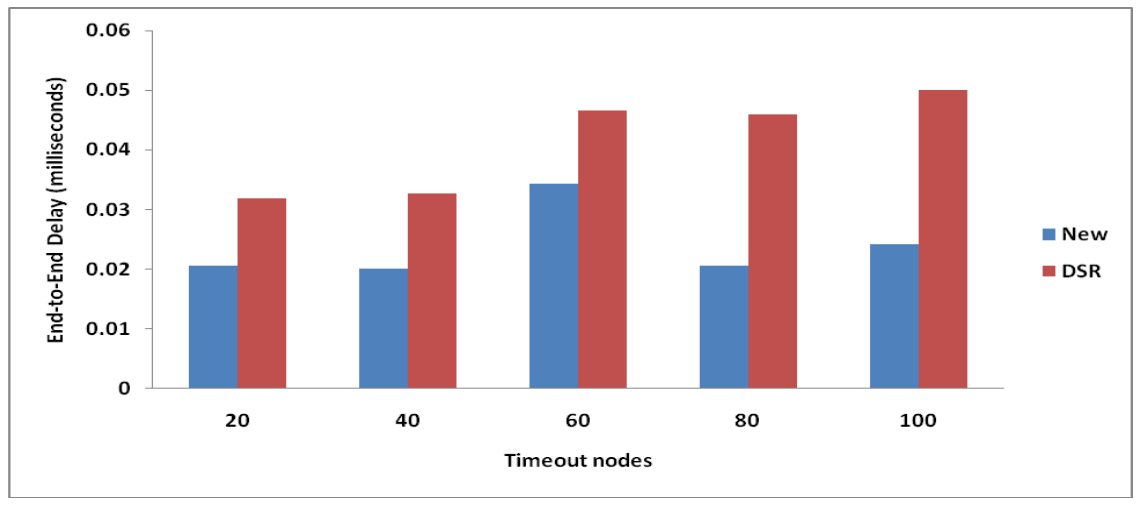

Fig.3. Throughput versus nodes pause time

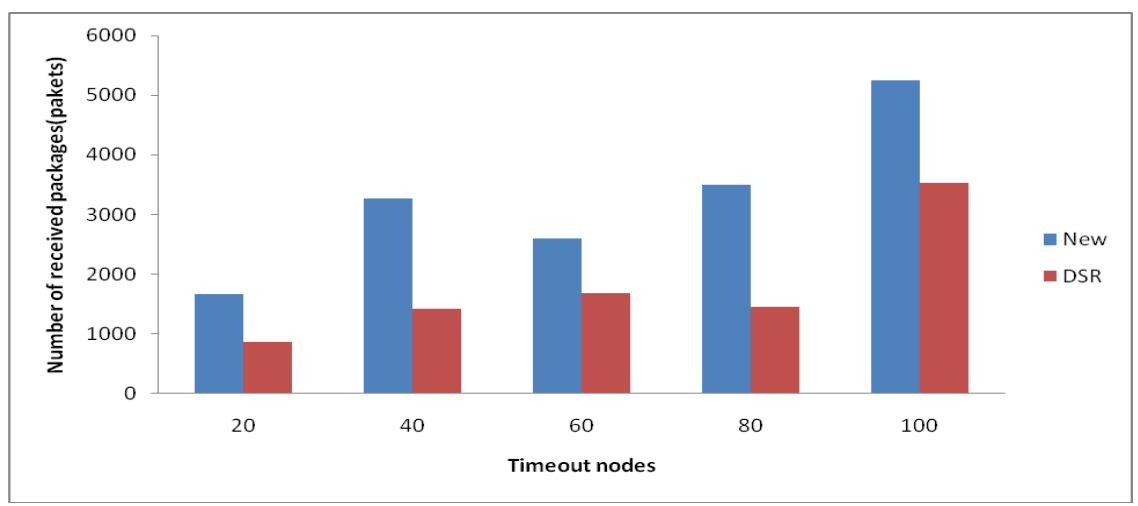

Fig.4. Number of received packets versus nodes speeds

In Fig. 2, by observing the average delay versus the stop time of the nodes, it is observed that at the same time, our proposed protocol has a lower latency than the DSR protocol. In Figure 3, by comparing the proposed protocol and the DSR protocol in terms of power efficiency, the result of our proposed protocol at identical times of 20 to 100 is better than the standard DSR protocol. Figure 4 also shows that the DSR protocol has a 
better performance than the DSR protocol in terms of the number of packets received at the destination.

\subsection{Second simulation scenario}

In this scenario, the number of nodes is considered variable. The simulation parameters for this scenario are shown in Table 3. The results for this scenario are shown in Figures 5 to 7.

Table. 2 Third simulation scenario parameters

\begin{tabular}{|c|c|}
\hline Parameters & Parameter Values \\
\hline Simulation space & $500 \times 500 \mathrm{~m}$ \\
\hline Simulation time & $900 \mathrm{~S}$ \\
\hline Routing protocol & DSR \\
\hline Nodes pause time & $10 \mathrm{~S}$ \\
\hline Nodes number & $15,20,30,40,50$ \\
\hline IFQ & Drop tail \\
\hline Queue capacity & 50 packets \\
\hline MAC layer & $802.11 \mathrm{~b}$ \\
\hline
\end{tabular}

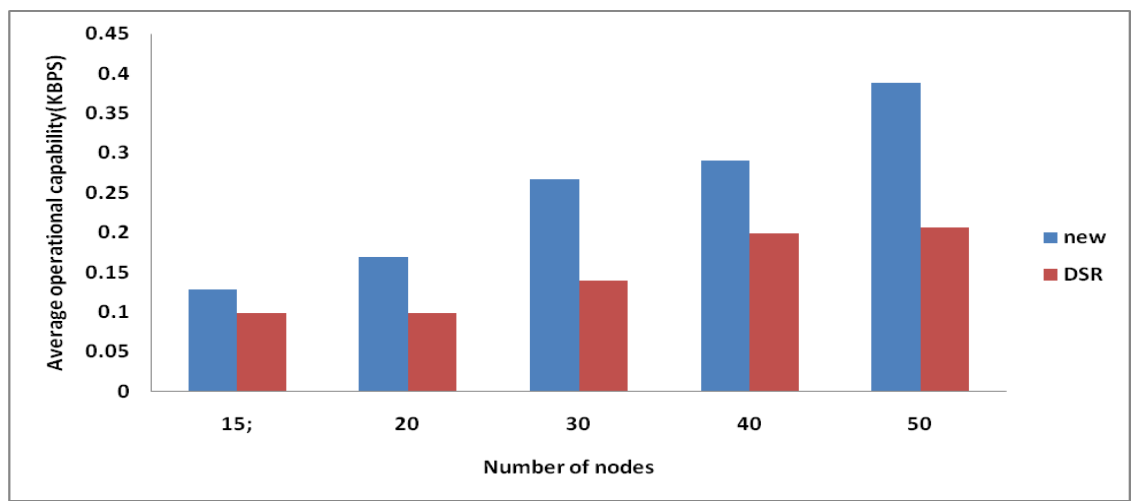

Fig.5.Average end to end delay versus number of nodes

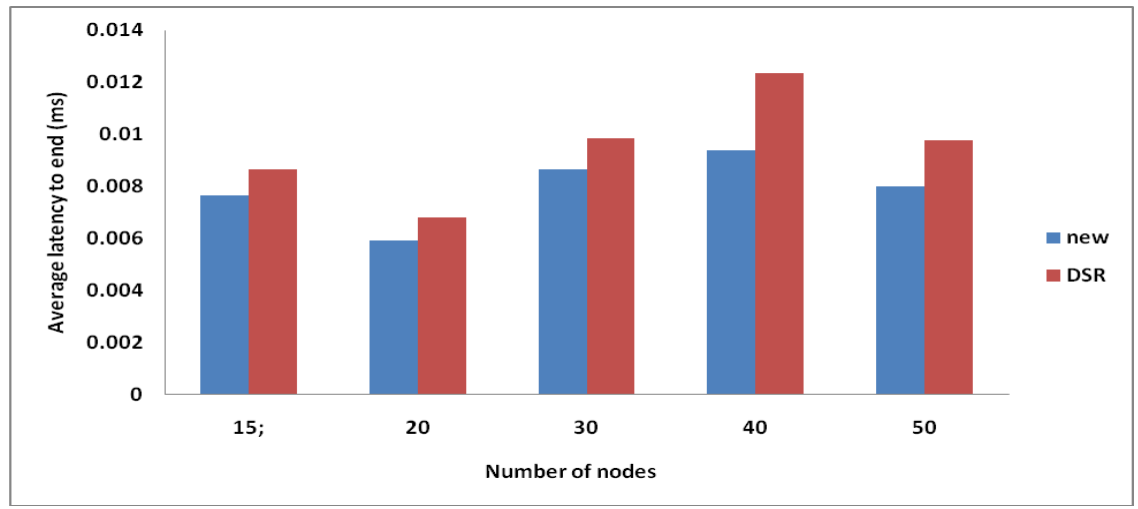

Fig.6. Average number of received packets versus number of nodes 


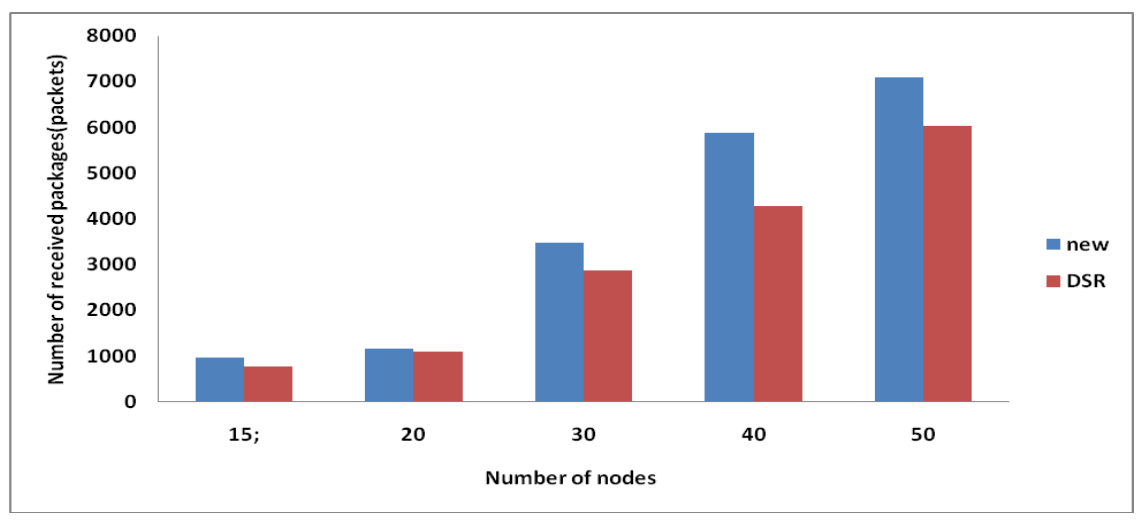

Fig.7. Average throughput versus number of nodes

As shown in the Figures 5 to 7, in this scenario, the ST-DSR protocol also performs better than the DSR protocol. The reason why our proposed protocol is better is that the protocol finds paths that are likely to take a long time. For this reason, data is sent from safer routes and the need for re-routing is reduced.

\section{Conclusions}

Given the high importance of routing in mobile ad hoc networks, in this paper we presented a new stable protocol for routing in mobile ad hoc networks. The protocol is based on the DSR protocol, and in discovering a path, a path is discovered that has sustainability and high quality in terms of the stability of the path links, the energy of the nodes and the path length. The simulation results in the NS-2 simulator indicate that the proposed protocol has a better performance than the proposed protocol.

\section{References}

[1] ElAttar H, Salwa H. Lifetime Maximization for Wireless Ad Hoc Networks Using Power Aware Routing. UKSIM European Symposium on Computer Modeling and Simulation, 2010

[2] Chlamtac I, Conti M, Jennifer J, Liu N. Mobile ad hoc networking: imperatives and challenges, Ad Hoc Networks (Vol. 1), 2003: 13-64

[3] Limin M, Weihuang F, Zhijiang X, Jiangxing Z, Jingyu H. A novel Ad hoc routing protocol based on mobility Prediction. Information Technology Journal (Vol. 7), 2008: 537-540.

[4] Chegin M, Fathy M. Optimized routing based on mobility prediction in wireless mobile Ad hoc networks for urban area. ITNG, 2008: 390-395.

[5] MohammadAl M, Mario K. Structured Peer-to-Peer overlay deployment on MANET: A survey. Computer Networks (Vol. 96), 2016: 29-47.

[6] Tang S, Li W, Kim J. Modeling and Evaluation of Traffic Flow and Availability for Mobile Ad hoc Networks. Proceedings of IEEE Wireless Communications and Networking Conference, 2006.

[7] Xiaoying Z, Anpalagan A, Lei G. Performance Improvement of Energy-Aware MANET Routing Algorithm Using Load-Balancing. 17th International Conference on Computational Science and Engineering (CSE), 2014.

[8] Ghafouri A, Ghasemi A, Hasani Ahangar M. R, A Power-based Method for Improving the ODMRP Protocol Performance in Mobile Ad-hoc Networks, International Journal of Wireless and Microwave Technologies (Vol. 2), 2018: 27-36. 
[9] Rajeshkumar V, Sivakumar P. Comparative Study of Routing Protocols in MANET. International Journal of Advanced Research in Computer and Communication Engineering (Vol. 2), 2013.

[10] Maan F, Mazhar N. MANET Routing Protocols vs. Mobility Models: A Performance Evaluation. 2011: $179-184$.

[11] Gyanappa W, Rajashekar B. A survey on hybrid routing mechanisms in mobile ad hoc Networks. Journal of Network and Computer Applications (Vol. 77), 2017: 48-63.

[12] Kale M, Ruchia A, Gupta S R, Prmit BR. An overview of manet ad hoc network. International journal of computer science and applications (Vol 6), 2013.

[13] Perkins CE. Ad hoc on-demand distance vector (AODV) routing. 1997.

[14] Tuteja A, Gujral R, Thalia S. Comparative Performance Analysis of DSDV, AODV and DSR Routing Protocols in MANET using NS2, in Proceedings of Conference, 2010 .

[15] Zafar M. Comprehensive experimental performance analysis of DSR, AODV and DSDV routing protocol for different metrics values with predefined constraints. International Journal of Information technology and computer science, 2014: 24-31.

[16] Shadi S.B, Marina D.V, Julian P, YushengJi T.L, Simon A. Energy efficient zone based routing protocol for MANETs. Ad Hoc Networks (Vol. 25), 2015: 16-37.

[17] Abeer G, EmanS. Power Aware Cooperation Enforcement MANET Routing Protocols. Procedia Computer Science (Vol. 73), 2015: 162-171.

[18] RagulRavi R, Jayanthi V. Energy Efficient Neighbor Coverage Protocol for Reducing Rebroadcast in MANET. Procedia Computer Science (Vol. 47), 2015: 417-423.

[19] Ravi G, Kashwan K. R. A new routing protocol for energy efficient mobile applications for ad hoc networks. Computers \& Electrical Engineering (Vol. 48), 2015: 77-85.

[20] Gaurav S, Vijay L, Gaur M. S, Swati T, Vijay R, Meenakshi T, Riti K. Multi-constraints link stable multicast routing protocol in MANETs. Ad Hoc Networks (Vol. 63), 2017: 115-128.

[21] Dilpreet K. Comparative analysis of AODV, OLSR, TORA, DSR and DSDV. International Journal of Computer network and information security, 2013: 39-46.

[22] Broch J, Johnson DB, Maltz DA. The dynamic source routing for mobile adhoc networks. 1998.

[23] Yiannis S. The impact of simulation duration on the performance of the OLSR, AODV and DSDV Protocols in a heavy-loaded Ad-hoc wireless mobile environment. First international conference on system informatics and modeling, IEEE 2014.

[24] Kundu P, Kashyap N, Securing DSR for Mobile Ad hoc Networks with Message Digest Algorithm, International Journal of Wireless and Microwave technologies (Vol. 5), 2016: 54-60.

\section{Authors' Profiles}

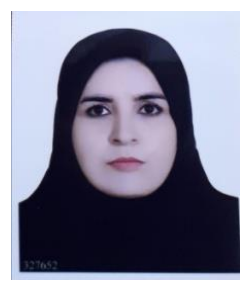

Golsum Najafi received her MSc degree in computer engineering from the Islamic Azad University of Germi Branch, Iran in 2017. Her research interest covers mobile ad hoc networks. 


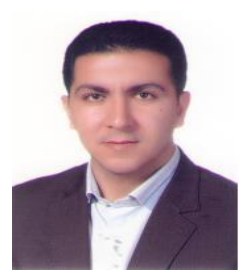

Sajjad Jahanbakhsh Gudakahriz recrived his MSc degree in computer engineering from Islamic Azad University of Qazvin branch, Iran in 2011 and now is PHD student in computer engineering in Islamic Azad University of Qazvin branch, Iran. He is faculty member in Islamic Azad University of Germi branch, Iran. His research interests cover mobile ad hoc networks and data mining.

How to cite this paper: Golsum Najafi, Sajjad Jahanbakhsh Gudakahriz," A Stable Routing Protocol based on DSR Protocol for Mobile Ad Hoc Networks", International Journal of Wireless and Microwave Technologies(IJWMT), Vol.8, No.3, pp. 14-22, 2018.DOI: 10.5815/ijwmt.2018.03.02 